\title{
PRACE World-Class Computational Facilities Ready for Polish Scientific Community
}

\author{
Elaborated by: \\ Mirosław Kupczyk, Norbert Meyer \\ Poznań Supercomputing and Networking Center \\ ul. Noskowskiego 10, 61-704 Poznań, Poland \\ e-mail:\{miron/meyer\}@man.poznan.pl
}

(Received: 30 September 2010; revised: 9 November 2010; published online: 23 November 2010)

\begin{abstract}
The article presents the current state-of-the-art of the European HPC ecosystem design and implementation. It covers the benefits for all ecosystem stakeholders: scientific end-users, operational staff, architecture vendors, owners of the computational facilities. The PRACE - Partnership for Advanced Computing In Europe initiative has started the preparation of the background towards the legal form of the related activities. It benefits in the creation of the PRACE non-profit association AISBL, registered in Brussels. During the implementation phase of PRACE, the scientific community has been given free and transparent access to the world-class supercomputing systems in Europe. Polish scientists and researchers may apply for the computational cycles.
\end{abstract}

Key words: PRACE, HPC ecosystem, PRACE AISBL, Petaflops systems, users support, European Research Infrastructure

\section{INTRODUCTION}

Over the past three decades, Computational Science and Engineering has steadily evolved into a key instrument for conducting research and development of new ideas, products and services. Known today as the Third Methodology, Computational Science and Engineering is considered to be of equal importance to theory and experiment. It is a key enabler of scientific and industrial progress and has transformed the modeling and prototyping of physical systems including those that are too large or too small to study directly. In all major economies worldwide its use is rapidly growing. This is leading to major advances in scientific research, driving industrial innovation and delivering strong societal impact. For its use and value to continue to grow, access to European supercomputers of the highest available performance must be made available as part of each country's HPC ecosystem.

The white paper "Scientific Case for Advanced Computing in Europe" [1] that was produced by the HET [2] in summer 2006, convincingly laid out the ambitious case for, and the huge potential of the field. Researchers from many disciplines of science and applied research contributed to the scientific case and stand ready to take advantage of the next generation of this innovative methodology. Furthermore, these activities strongly influenced the ESFRI [3] recommendations for new
European research infrastructures made in December 2006. In summary, Computational Science and Engineering fuelled by access to the best supercomputers was demonstrated to have the potential to profoundly influence science and engineering through its ability to simulate highly complex phenomena from first principles.

Here we present the current status of the European HPC ecosystem design and implementation. It covers the benefits for all ecosystem stakeholders: scientific end-users, operational staff, architecture vendors, owners of the computational facilities. The project called PRACE (Partnership for Advanced Computing In Europe) [4] had started the preparation of the background towards the legal form of the related activities. It benefits in the creation of the PRACE non-profit association AISBL, registered in Brussels. During the implementation phase of the PRACE1IP (PRACE - First Implementation Phase Project) [5], the scientific community has been given free and transparent access to the world-class supercomputing systems in Europe. Polish scientists and researchers may apply for the computational cycles.

\section{THE PREREQUISITES}

A recent European publication in Science [6] exemplifies this striking progress in the field of Computational 
Science and Engineering: today the most advanced quantum chemical methods together with the fastest supercomputers allow for detailed simulation of a prototypical surface reaction: $\mathrm{H}_{2}$ dissociation on $\mathrm{Cu}$ with chemical accuracy. This specific example demonstrates that supercomputing has become directly applicable to problems that are highly relevant for the future of our society; in this case the hydrogen energy economy and the catalysis for most of the synthetic materials. As mentioned above, the HET Scientific Case documents this progress for a large variety of fields and shows both recent achievements and its future perspectives.

Several groups have made prospective predictions and presented roadmaps for future research, spanning the next decade and considering the availability of supercomputers up to exascale performance. The following examples show fields that have already reached a high level through the use of computational science and where further leadership of European science and engineering will very much benefit from or even critically depend on the availability of the most advances computing resources at the Tier-0-level:

- Provide models for the prediction of materials properties at various scales, based on ab initio basic principles (i.e. with decreasing reliance on intermediate empirical models). Of utmost importance in fields where experiments are impossible to verify the intermediate models.

- Provide predictive models of very complex systems (climatology, social behaviour).

- Provide the basis for computational and model-based design in biotechnology and nanotechnology, permitting the elucidation of complex interaction at the nano-scale and nano-scale design.

- Make personalised medicine feasible. Examples are optimised surgery, adaptation of drugs protocols to the individual genome. In both cases, the "time to solution" constraint is essential, albeit very different.

- Contribute to fighting pandemic virus infections in "real time".

- Well beyond real-time simulation of water management issues ("what-if cases in emergency situations").

- Permit extensive engineering optimisation of complex designs (aerospace, energy), and consideration of tolerance margins in products and processes, verification and validation of engineering and scientific models without experiments.

The list could be continued by many examples, from basic research in astrophysics and astronomy, biology, chemistry, condensed matter, geophysics, or nuclear physics where Europe often is in a leadership position. The presented examples also show an increasing tendency for using supercomputers for "real time" prediction, either in exceptional cases or as a repetitive routine activity. This in turn brings about challenging requirements in resource allocation and scheduling and the interoperability with the "problem related" ecosystem of resources and teams.

A general conclusion is that the continuation of leadership and further progress of Europeans computational science and engineering strongly depend on the availability of computing resources on the highest performance level, world-class support structures, and peer-reviewed access for scientists from all over Europe.

The PRACE-1IP project will provide essential scientific, organisational and technical contributions to the PRACE RI. The future HPC ecosystem will provide European with Tier- 0 facilities at the highest performance level and will enable access to and interoperation with national and regional Tier-1 facilities. The latter in turn will integrate regional and topical Tier- 2 centres. The competence and support teams on all levels will closely interact. The HPC ecosystem will allow access to the most innovative modeling and simulation codes for all European scientists and will foster their further development and evolution.

\section{GOAL}

The main objective of the PRACE-RI is to provide the communities with a leading edge supercomputing infrastructure and corresponding world-class support for its most effective use. This infrastructure will guarantee European scientists and researchers access to the best facilities in a highly competitive world. Furthermore, it will give Europe the autonomy and independence to decide on suitable directions to address the many social issues we face worldwide today based on our own verifiable research results from our own e-Infrastructures.

On the one hand, the PRACE-RI will focus on the top of the computing pyramid (Fig. 1), whilst on the other hand, it will lead the integration of its infrastructure within the HPC-ecosystem formed by the national and regional centers represented by the PRACE-RI partners who themselves are in close contact with local regional centers and Grids or Clouds. In particular, PRACE benefits from the results of the European DEISA [7] project and continue the successful work that DEISA has started by providing access to national and regional systems for European scientists and engineers. The vision is that leading edge computing at the top of the European computational infrastructure pyramid builds upon the other layers, giving value to the whole in turn by permitting the infrastructure to tackle and solve the major scientific challenges. 


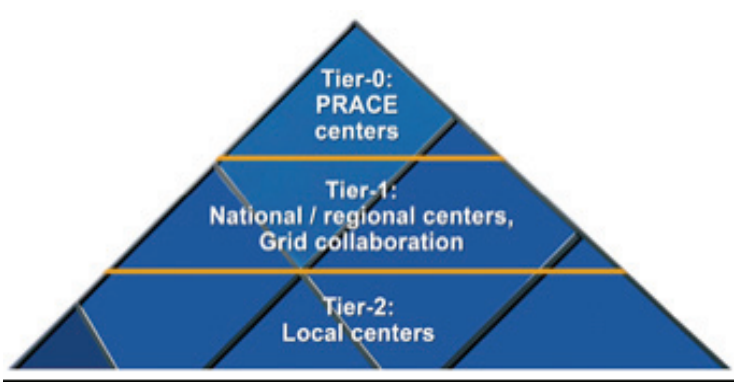

Fig. 1. PRACE pyramid of sites' performance

\section{THE REALIZATIONS}

Building a world-class pan-European High Performance Computing (HPC) Service is a highly ambitious undertaking that involves governments, funding agencies, centres capable to host and manage the supercomputers, and the scientific and industrial user communities with leading edge applications.

In contrast to Research Infrastructures that focus on a single scientific instrument an HPC Infrastructure has unique characteristics: today's supercomputers will no longer be competitive in two to three years. This requires a periodic renewal of the tier- 0 systems and a continuous upgrade of the infrastructure. Furthermore, novel architectures and system designs will be created by the vendors for leadership systems. At any given time there will be between three and five different systems each of them serving a particular user community best. This fact mandates a distributed Research Infrastructure, since no single site can host all the necessary systems because of floor space, power, and cooling demands.

In the preparatory phase, PRACE created the prerequisites for such a pan-European HPC service satisfying the objectives mentioned above and to move into the implementation phase in 2010 (PRACE-1IP) In detail, the following were elaborated:

- Selecting of an appropriate legal form, the definition of its statute, its governance in relation with the partners, the European Commission and the scientific users. The targets are signature ready contracts for the creation of a permanent European HPC service.

- Establishing funding strategies and usage models to ensure sustained funding.

- Defining a peer review process to support leading edge science and optimal use of the resources ensuring open, fair and unbiased access to the Research Infrastructure.

- Defining a consistent operational model across the distributed tier-0 sites.
- Managing the PRACE project using principles suitable for the permanent infrastructure.

- Disseminating of project achievements, establishing and maintaining links with selected industries both as users of capability systems, technology providers, and creators of new technologies with HPC, and training of potential users.

In addition to the legal work and the creation of the administrative structures, PRACE performed accompanying technical work to prepare for the production phase:

- Providing tools for consistent management of the tier- 0 systems and for the integration of the tier- 0 infrastructure into the European HPC ecosystem.

- Deployment of prototypes of leadership class systems at selected sites that are likely to become productions level systems in 2010.

- Porting, optimising, and petascaling selected applications to ready them for production on the tier- 0 systems. Creating benchmark suites.

- Defining a consistent procurement strategy, associated technical specification, selection criteria for the current and future generations of HPC services, and the requirements for the physical infrastructure to host tier-0 systems.

- Starting a permanent process of technology evaluation to transform user requirements into specifications for future leadership class systems. This process that will last throughout the lifetime of the infrastructure will be done in close relations with vendors of components, systems, and software.

\section{MAIN ACHIEVEMENTS}

\section{V.1. PRACE AISBL (Association Internationale Sans But Lucratif)}

PRACE, the Partnership for Advanced Computing in Europe, has been established as a non-profit association (AISBL) under Belgian law with its seat in Brussels. Founding members are: Austria, Bulgaria, Finland, France, Germany, Greece, Ireland, Italy, the Netherlands, Poland, Portugal, Serbia, Spain, Switzerland, Turkey, and the United Kingdom. Deed signed in Brussels: April 23rd, 2010. The Polish representative in PRACE is Poznań Supercomputing and Networking Center (PSNC), as a socalled 'non-hosting partner'.

The main excerpts from the Statuses of PRACE AISBL are:

- Use of PRACE is free-of-charge for European scientific communities 
- Use of PRACE is free-of-charges for companies that make public the results of the research project

- Access based on scientific quality and their need to use tier-0 system

- Renewal of Tier-0 systems every 2-3 years required

- Binding commitments for $400 \mathrm{M} €$ from France, Germany, Italy and Spain over 5 years.

- Investments of 500-600 M€ next 5 years will create a critical mass for strengthening the European HPC industry

- Capability to provide independent access to this key technology

- Access to world-class HPC systems as a competitive advantage for European science and industry

- A European HPC Service as part of the European Research Area

- Access to world-class HPC systems for the best researchers from all European countries

- PRACE Headquarters funded by all members

Summing up, PRACE will maintain a pan-European HPC service consisting of up to six top of the line leadership systems (Tier-0) well integrated into the European HPC ecosystem. Each system will provide computing power of several Petaflop/s in midterm. On the longer term (2019) Exaflop/s (one quintillion) computing power will be targeted by PRACE. Users will be supported by experts in porting, scaling, and optimizing applications to novel, highly parallel computer architectures. An in-depth training program accompanies the PRACE offering teaching scientists and students how to best exploit the unprecedented capabilities of the systems. A scientific steering committee will provide advice to PRACE and operate alongside a bespoke peer review process through which access to the Tier- 0 resources will be granted based on scientific excellence.

\section{V.2. PRACE Prototypes}

One of the outcomes of the PRACE project is prototype testing for supporting decisions for the installation of the future HPC Petascale systems in Europe. In this context some pre-production prototypes have been installed at host centres of PRACE partners, and researchers are now invited to test their codes in these systems:

- BSC (Barcelona Supercomputing Center, Spain), installs a hybrid prototype combining IBM Cell and Power6 processors. The Cell processors are used for computation and the Power6 processors for service.

- CEA (French Atomic Energy Commission, France) and FZJ (Forschungszentrum Jülich, Germany) jointly use Intel Nehalem/Xeon processors in their systems. Two shared-memory multiprocessors (thin node clusters) are distributed over the two sites; a prototype produced by BULL at CEA and a larger system of the same architecture at FZJ.

- CSC (The Finnish IT Center for Science, Finland) and CSCS (Swiss National Supercomputing Centre, Switzerland) jointly evaluate the Cray XT5 architecture. This Massively Parallel Processing (MPP) prototype is installed at CSC's facilities.

- FZJ provides IBM BlueGene/P system, as a Massively Parallel Processing prototype.

- HLRS (High Performance Computing Center Stuttgart, Germany) offers a NEC SX-9 and an x86 based cluster as a hybrid prototype.

- NCF (Netherlands Computing Facilities Foundation, The Netherlands) evaluated the IBM Power6 architecture, a shared-memory multiprocessor (fat node cluster). This prototype is installed in SARA Computing and Networking Services facilities in Amsterdam.

\section{V.3. Who can apply for PRACE prototype access}

Proposals from academia and industry are eligible, as long as the organisation of the project leader is homed in Europe (or a PRACE initiative country). Depending on the system vendor, researchers from some countries cannot be granted access due to export regulations. The general conditions for prototype access are as follows:

- Prototypes are intended for testing and not for standard production work.

- As a general rule application support (e.g. code development, optimisation, parallelisation, etc.) during prototype testing is limited.

- Prototype access is granted after technical review by the PRACE centre hosting the prototype.

- The maximum project duration is 3 months.

- Applicants must agree that applicants' names and affiliations, as well as a summary of the project purpose and the results achieved during prototype testing, may be made publicly available in the PRACE website and may be used in PRACE deliverables and other PRACE documents.

- Applicants must agree to summarise the results of the project in an end report to be sent to the PRACE centre hosting the prototype not later than 1 month after the project conclusion.

- The project leader is responsible for signing and fulfilling the usage agreements issued by the PRACE centre hosting the prototype.

- Proposals are accessed by the PRACE Prototype Access Committee with representatives from the prototype host centre. 
- Applicants have the right to reply to the granting decisions. Application replies will be handled by the PRACE Technical Board.

One of the main goals of the PRACE project was investigating petascale prototypes and carrying out research into scaling and optimising applications on petascaling machines. In order to access the PRACE prototypes, the application will be reviewed using a number of criteria. Priority will be given to applications that are on different applications to those already being investigated by PRACE [8]. There is a limited amount of time available in the prototype systems so access will only be given to those proposals that aim to investigate performance of an application, scale the application or carry out optimisations. There should be no production runs and as such there will be no consideration given to any potential new scientific results obtained.

\section{V.4. Production System User Access ("call for proposals")}

PRACE AISBL (Partnership for Advanced Computing in Europe) allows researchers from across Europe to apply for time on high-performance computers from a series of hosting nations via a central peer review process. This call is the first PRACE Regular call for Project access, inviting applications for high-end (Tier-0) computing resources to carry out projects which have high scientific quality and impact. Allocation is for 1 year.

The PRACE Regular call is intended for large-scale projects of high scientific quality and for which a significant impact at the European and international level is anticipated. High scalability of the code (at least 8000 compute cores) must be demonstrated. Proposals for project access must be ready to run. The projects must demonstrate scientific excellence and should cover topics of major relevance for European research. They should also include elements of novelty, transformative aspects, have a recognised scientific impact and include a dissemination plan. Possible practical and timely applications resulting from the project are also desirable. The projects should also demonstrate the possibility of achieving results which will be publishable in journals of recognised scientific impact.

The projects will be technically and scientifically peerreviewed by recognised experts. Applicants have the right to reply to the comments of the reviewers. The proposals, together with the reviewers' reports and the applicants' responses, will be analysed by the Prioritisation Panel who will produce the final ranking list. Proposals will be awarded by moving down the ranking list in order until quality or resources run out. If necessary, the Prioritisation
Panel may agree on a quality cut-off threshold. Proposals ranked under the cut-off threshold will not be awarded even if there is resource left on the machine [9].

The HPC system available to researchers through the 1-st PRACE Regular call is the IBM Blue Gene/P JUGENE - hosted by GCS in Jülich, Germany. The maximum number of compute cores available at JUGENE is 294912 . The total available capacity in this call was 360 million compute core hours. All proposals must be submitted via the PRACE website at [9].

\section{SUPERCOMPUTING TECHNOLOGY}

The rapid pace of improvement of microprocessors has made very cost-effective computers available for numerous kinds of applications. However, the requirements of leading-edge scientific applications are rapidly outgrowing the technological progress that has an innovation cycle of three to four years. Such applications demand very long execution times even on today's largest supercomputers. Since, during the last five years, the previously quite continuous progression of processor frequencies has come to an end, the chip industry resorts to multi- and many-core processors. On the one hand, this approach still allows following the progress in performance characterized by Moore's law. On the other hand, the ensuing extremely high parallelism poses new, extreme challenges: machines are built with hundreds of thousands of processor cores, new hybrid architectures emerge that use GPUs as coprocessors. Hardware, parallelism is spatially constrained, because many applications need to exchange between processors at a level that the speed of light becomes the limiting barrier in the computational process. A further ever more severe barrier arises from the physical constraints through power consumption and cooling requirements of the hardware. All this has made leading edge supercomputers very different from earlier computing systems used for scientific, engineering or industrial applications - they present increasing challenges at all levels from design to procurement and operations.

Countries like the United States, Japan and China are pushing technological development, performance and the application of supercomputers very aggressively, recognising that these resources are essential to national success in science, engineering, and economy. Currently, computers of one Petaflop/s are state-of-the-art. The next long-term milestone is defined by the Exaflop/s barrier to be reached around the end of the next decade (2020).

In Europe, the PRACE RI, with the objective to provide sustained world-class computational capabilities to the 
European scientists, is directly confronted with these enormous technological challenges. They span current engineering and scientific concepts concerning memory hierarchy, data transfers at all levels from the chip to the system, power consumption and heat dissipation and reliability. In order to meet these challenges, the integration of novel designs at all levels, from circuits to systems; from system software to scientific application codes is essential. The PRACE-1IP will work with suppliers to gain access to promising architectural innovations, with the intention to field fully operable mature leading edge systems. This will be achieved by investing in technology evaluation, prototyping, training of application designers, and developing new or improved applications. The PRACE-1IP project involves several work packages addressing novel architectural designs for computers and applications.

\section{SUMMARY}

The approach taken in the PRACE Preparatory Phase project has been found very effective. It involved the deployment of prototypes with innovative hardware, technology and software tools, both at a stage close to market availability as well as forward looking activities. It was enhanced by sharing expertise across European teams and centres to a hitherto unprecedented level. PRACE and the PRACE 1IP project will provide European scientists and researchers from academia and industry with an environ- ment that enables them to be internationally competitive today and in the future.

\section{References}

[1] The Scientific Case for a European Super Computing Infrastructure, http://www.hpcineuropetaskforce.eu/files/"Scientific case for European HPC infrastructure HET.pdf", 2006.

[2] HET - HPC in Europe Taskforce, homepage: http://www.hpcineuropetaskforce.eu

[3] ESFRI - European Strategy Forum on Research Infrastructures, homepage: http://ec.europa.eu/research/infrastructures/index_en.cfm?p $\mathrm{g}=\mathrm{esfri}$

[4] PRACE - Partnership for Advanced Computing In Europe project, FP7/2007-2011, RI-211528, homepage: http://www.prace-project.eu

[5] PRACE-1IP - PRACE - First Implementation Phase Project, FP7 contract No 261557, homepage: http://www.prace-ri.eu/

[6] Chemically accurate simulation of a prototypical surface reaction: $\mathrm{H} 2$ dissociation on $\mathrm{Cu}(111)$. C. Díaz, E. Pijper, R.A. Olsen, H.F. Busnengo, D.J. Auerbach, G.J. Kroes. Science 6 November 2009

[7] DEISA - Distributed European Infrastructure for Supercomputing Applications, FP7 contract No RI-222919, homepage: http://www.deisa.eu/

[8] A. D. Simpson, M. Bull, J. Hill, Identification and Categorisation of Applications and Initial Benchmarks Suite, http://www.praceproject.eu/documents/Identification_and_Categorisatio_of Applications_and_Initial_Benchmark_Suite_final.pdf

[9] PRACE peer review process, www.prace-project.eu/hpcaccess
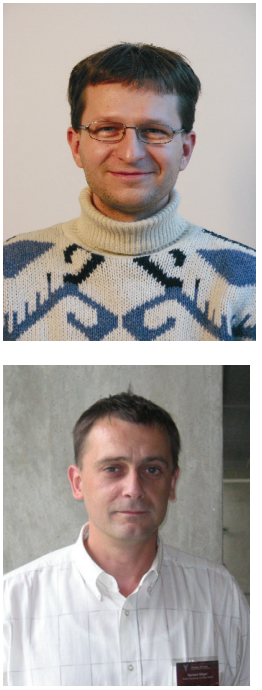

MirosŁaw KuPCZYK received M.Sc. degree in Computer Science from the Poznań University of Technology, Distributed Computer Systems specialization. Currently he is employed as HPC specialist at the Supercomputing Department in Poznań Supercomputing and Networking Center (PSNC). His research interests concern resource management, parallel programming and processing, graph algorithms. Since 1998 MK has been responsible for putting into practice workload management system on PSNC production machines. He has been involved in several grid projects (PL-GRID, PRACE, BalticGrid, EGEE, FaultTolerant Grids (France Telecom), etc.). He is an author and co-author of several reports and papers in scientific journals and conference proceedings.

Dr. NoRbert Meyer is currently the head of the Supercomputing Department in Poznan Supercomputing and Networking Center (http://www.man.poznan.pl). His research interests concern resource management in GRID environment, GRID accounting, data management, technology of development graphical user interfaces and network security, mainly in the aspects of connecting independent, geographically distant Grid domains. NM conceived the idea of connecting Polish supercomputing centres, vision of dedicated application servers and distributed storage infrastructure. He is the author and co-author of $60+$ conference papers and articles in international journals, member of programme committees of international conferences related high performance computing and distributed computing. He was the leader of RINGrid EU project, currently leading the EU DORII project. 